\title{
Public Health and Mass Gathering Events: Assessing Need for Surveillance in Wales, UK
}

\author{
Priyanka Parmar ${ }^{1}$ and Daniel RH Thomas ${ }^{\star 2}$ \\ ${ }^{1}$ Immunisation Technical Support Unit, Public Health Foundation of India, Gurgaon, India; ${ }^{2}$ Public Health Wales, UK
}

\section{Objective}

To identify the need of surveillance during mass gathering events in Wales by identifying a causal relationship between public health and characteristics of a mass gathering event grounded on risk assessment.

\section{Introduction}

Mass gathering is defined by WHO as - "More than specified number of persons (which can be as few as 1000 persons to more than 25000) at a specific location for a specific purpose (social function, large public event or sports competition) for a defined period of time" . Mass gatherings are considered to have the potential for public health incidents, outbreaks and casualties attributed to the inevitable overcrowding in a place. Because populations are increasingly mobile, and more able to attend large gatherings, the risk for outbreaks of infectious diseases among a susceptible population has increased, and a substantial responsibility is placed on health services if outbreaks occur.

Currently, there are no existing programmes of surveillance for mass gathering events in Wales. Although a variety of surveillance system exist, no systematic process is established to gather the information from important large events and collect into a common database for future reference and to study the impact. The number of people attending these gatherings in Wales range from few thousands to 250,000 .

\section{Methods}

An intensive literature review was carried out to identify risk variables in mass gathering events. Based on identified risks a 'risk assessment tool' was developed for the mass gathering events. The risk variables are given a score according to its known risk level. The maximum total risk level score of an event according to tool can be 27 and lowest 2. All the identified mass gathering events in Wales were assessed according to this tool. A web based semi structured survey to identify public health issues during mass gatherings in Wales was designed on the NHS website for local public health officials involved in preparedness of mass gatherings.

\section{Results}

The assessment tool was observed consistent with the other international events in the world when their risk levels were compared. Mass gathering events in Wales were identified as low and medium risks. High risk events that were identified by tool were also identified in the survey. Alcohol and drug abuse, food safety, accidents, young adults and poor weather (rain) were the factors mainly highlighted as high risk variables in the survey. $75 \%$ of the respondents mentioned prior preventive measures undertaken during mass gatherings. Follow up of the mass gathering events and surveillance were noticed to be absent. Surveillance is a part of ongoing epidemiological surveillance in different health sectors but mass gathering events are only monitored if any serious outbreak occurs otherwise the routine measures are followed.

\section{Conclusions}

The risk level depends on the dose of each risk variable in the event on the attending individuals. The need for a continuous surveillance is associated to the risk level of the event: High - Requires surveillance and action to be taken to reduce consequences; Moderate - Requires regular monitoring and preparedness ; Low - Requires routine measures. Thoroughly planned and prepared events usually show fewer chances of any public health issues. Various factors have been identified as high risks (weather, age group of attendees and alcohol and drug abuse) which necessitate the need of monitoring the events. Most of the studies were found to support enhanced surveillance system during large national and international mass gathering events, which helps them to see the outcome of the surveillance and impact of the mass gathering. Enhancing the current surveillance system to establish a collaborated department would require less resources to monitor events and for training in emergency conditions. A detailed planning of deliveries during future events, adequate staffing, defined funding and resources and cost effectiveness should be implemented.

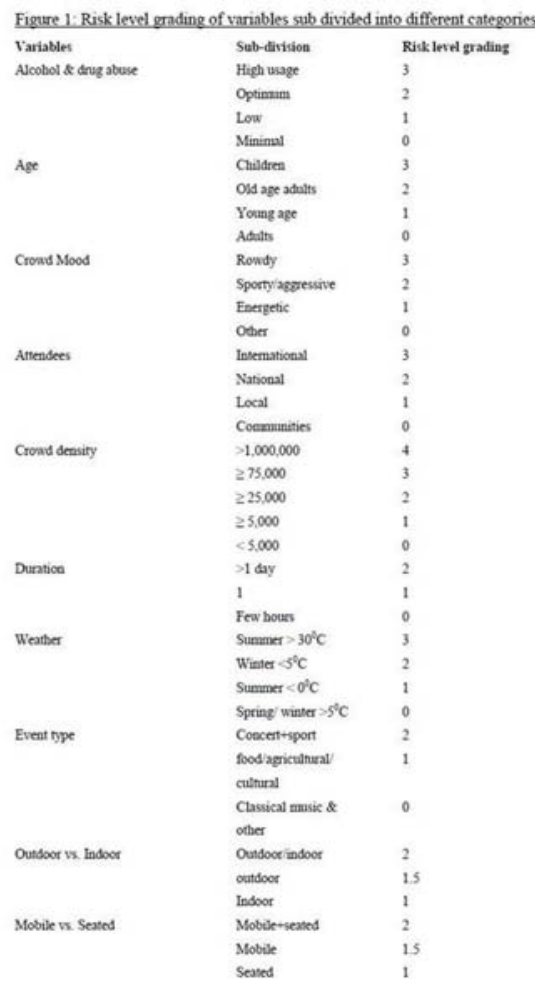

Keywords

Mass gatherings; Mass gathering events; Surveillance; risk assessment

\section{References}

1. Communicable disease alert and response for mass gathering. Key Considerations. World Health Organisation. June 2008. 7 p.

*Daniel RH Thomas

E-mail: daniel,thomas@wales.nhs.uk 